\title{
Rythme et temps musical dans le théâtre Tudor
}

\section{Francis Guinle}

\section{(Q) OpenEdition \\ Journals}

Édition électronique

URL : http://journals.openedition.org/shakespeare/513

DOI : 10.4000/shakespeare.513

ISSN : 2271-6424

Éditeur

Société Française Shakespeare

Édition imprimée

Date de publication : 1 novembre 1989

Pagination : 43-52

Référence électronique

Francis Guinle, "Rythme et temps musical dans le théâtre Tudor ", Actes des congrès de la Société française Shakespeare [En ligne], 6 | 1989, mis en ligne le 01 janvier 2007, consulté le 27 avril 2019. URL : http://journals.openedition.org/shakespeare/513; DOI : 10.4000/shakespeare.513 


\section{SOCIETE FRANCAISE SHAKESPEARE}

\section{Actes du Congrès - 1984}

\section{LIEU ET TEMPS}

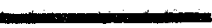

Directeur de la publication Jean FUZIER 


\section{Rythme et temps musical dans le théâtre Tudor}

La structure d'une pièce de théâtre, comme celle de toute forme d'art, résulte du désir de construire une composition harmonieuse, selon les critères particuliers d'une époque. L'harmonie devient alors une expression du Beau, sinon du Sublime. La rencontre des formes artistiques, pour être réussie, doit se produire en fonction de cette harmonie. La volonté de réaliser ce que Wilfrid Mellers appelle, dans un livre qui porte ce titre, "Harmonious Meetings "1, paraît évidente à tout familier du théâtre Tudor. La place importante qu'y tient l'élément musical, et l'utilisation constante du vocabulaire musical, en sont deux caractéristiques fondamentales.

L'aspect du temps qui nous retient ici s'apparente à la notion de temps selon le sens musical du terme. Il se présente sous deux formes: l'unité de temps, par laquelle une phrase est mesurée, et dont les variations et la périodicité engendrent le rỵthme de cette phrase, et la pulsation, le tactus, temps fort qui crée sa dynamique. Sur ce double aspect du temps, déterminant rythme et dynamique, s'articule le schéma des moralités et des interludes. Le parallèle entre composition musicale et pièce frappe, et l'on retrouve, dans les unes et les autres, le même enchaînement de sons et de silences, la 
même succession de temps forts et de temps faibles. Par temps fort, je n'entends pas le terme technique de solfège, lié à l'idée récente de mesure (mesure à deux temps, à trois temps, etc), mais simplement des moments musicaux forts, tels qu'un changement de tonalité, une modulation, la présentation d'un nouveau thème... Le développement d'un thème dans une tonalité, après une modulation, ou dans une variation, conduit la composition d'un temps fort à un autre, les phrases musicales se déroulant à partir d'eux. La structure théâtrale possède cette dynamique qui alterne temps forts et temps faibles, et s'organise autour des moments qui font basculer l'ensemble, semblables à un changement de tonalité; comme dans une composition polyphonique, on y observe des contrepoints, des thèmes secondaires, et des silences de l'action. En même temps, l'alternance de scènes où le dialogue est constitué d'échanges brefs et rapides, et de scènes où, se voulant didactique, il impose de longues leçons morales et religieuses, donne le rythme particulier de ces pièces, rythme que l'on retrouve jusque dans la période élisabéthaine, surtout dans le théâtre de John Lyly.

Mais ceci n'est pas le seul aspect musical du temps dans les pièces du théâtre Tudor. Le temps de la réalité, même court-circuité pour le faire coïncider avec le temps de la représentation, s'efface le plus souvent devant un autre temps, symbolique, marqué par un rituel musical. Ce temps du rituel persiste dans les pièces élisabéthaines, alors même qu'elles semblent présenter une forme du temps de la réalité.

La pulsation dramatique des pièces du théâtre Tudor, qu'elles soient du début de la période ou de l'époque élisabéthaine, destinées à une troupe d'adultes itinérante ou fixe, ou encore à une troupe d'enfants, s'exprime souvent par l'utilisation de la musique. Les temps forts varient selon le contexte, bien que l'on puisse trouver un motif commun dans le thème central de l'initiation, qui inscrit l'élément musical dans le cadre du rituel.

Les temps forts peuvent marquer l'entrée d'un nouveau personnage, sa sortie, ou celle d'un groupe, à la suite d'un épisode important, un accord entre les personnages quant à la ligne de conduite à suivre, un tournant décisif de l'action, une mort, une résurrection, un dénouement heureux ou malheureux. A chaque étape, la musique, instrumentale ou vocale, ponctue et rythme ce qui n'est plus perçu 
comme temps de la réalité, et dépasse la simple perception du temps de la représentation qui se trouve ainsi ritualisé.

Selon le schéma des moralités, le protagoniste se voit propulsé hors du temps réel en suivant les vices, et ainsi projeté dans le temps et le lieu de la discorde : la représentation verbale de l'enfer en témoigne, ainsi que la mention des lieux de débauche (par exemple la taverne vers laquelle les vices entraînent le protagoniste avec une chanson).

Le premier temps fort intervient à l'entrée du personnage central. Youth, dans Lusty Juventus, se présente en chanson :

In a herber grene, a slepe where as I lay

The byrdes sang swete in the myddes of the day,

I dreamed fast of mirth and play,

In youth is pleasure, in youth is pleasure.

Methought as I walked stil to and fro, And from her company I could not go,

But when I waked it was not so,

In youth is pleasure, in youth is pleasure.

Therefore my hart is surely pyght,

Of her alone to have a sight,

Which is my joy and hartes delyght

In youth is pleasure, in youth in pleasure. $\left(1\right.$ l. 38-49) ${ }^{2}$

Notons que la chanson indique le temps de l'année (" herber grene "), et celui du jour ("myddes of the day"), annonçant la thématique de la jeunesse et de la force de l'âge, et situant ainsi la pièce dans son cadre symbolique.

La soumission aux vices s'exprime par un accord, et devient effective au moment où le protagoniste se joint à eux pour une chanson à plusieurs voix :

Hypocrisy: Now I beshrew his herte, that to that wil not agree But yet because the time shal not seme very longe Or eare we depart, let us have a mery song. (1 1. 885-887) 
Le temps qui s'écoule, entre l'entrée du personnage central et sa soumission aux vices, est proportionnel à sa résistance. Youth (Lust) est déjà enclin au vice, et il l'annonce clairement dans sa chanson d'entrée; sa conversion n'est qu'une formalité, un rite auquel il doit participer, et la chanson devient la signature du pacte :

Why should not youth fulfil his owne minde

As the course of nature doth him bynde

Is not every thyng ordained to do his kinde?

Report me to you, report me to you.

Do not the floures sprynge freshe and gaye, Pleasaunt and swete in the month of May?

And when their time cometh they fayd awaye, Report me to you, report me to you.

Be not the trees in wynter bare

Like unto their kynde, such they are,

And when they springe their fruites declare

Report me to you, report me to you

What should youth do, with the fruites of age,

But live in pleasure in hys passage,

For when age commeth his luste wyll swage

Report me to you, report me to you

La métaphore continue, la chanson illustre la doctrine du carpe diem, et se sert de l'allégorie et de la généralisation pour ritualiser la soumission de Youth qui devient un initié.

Toutes les pièces ne suivent pas nécessairement ce schéma, mais beaucoup s'en inspirent plus ou moins. Les "Wit plays " ${ }^{3}$ proposent aussi une conception du temps et de l'action ritualisés, ajoutant un élément narratif sous forme de voyage initiatique, élément qui prend de plus en plus d'importance dans le développement du théâtre Tudor, pour parfois masquer l'influence des moralités dans les pièces plus tardives.

Le voyage de Wit, sa rencontre avec Tediousness, son combat, sa mort, sa résurrection, sa chute dans l'oisiveté, son second combat, sa 
victoire, et son union avec Science, tous ces événements se succèdent à un rythme qui n'a rien à voir avec le temps réel, et qui symbolise un trajet initiatique, des étapes à franchir, et non pas un écoulement du temps. Les temps forts de ce trajet sont ponctués musicalement. Ainsi la résurrection, puis la chute dans l'oisiveté sont accompagnées, dans les trois pièces, de chants ou de danses. L'aspect rituel de ces scènes se trouve accentué par l'épisode central du combat et de la résurrection, épisode emprunté aux pièces folkloriques de St George, connues sous le nom de "Mummers' Plays", elles-mêmes représentant la récupération d'un rite païen au moment du solstice d'hiver, donnant à ces pièces une dimension cosmique, les faisant surgir, en quelque sorte, de la nuit des temps, et les plaçant sous le triple signe de la fête, la commémoration, et du rite.

L'élément allégorique des pièces du début de la période se transforme, évolue, mais ne disparaît jamais complètement. Même lorsque une forme de réalisme s'y infiltre, le besoin de continuer à rythmer les pièces, à les faire entrer dans un temps musical, demeure. Les pages des comédies de cour héritent de certaines fonctions des vices des moralités, mais leur chant ne fonctionne plus comme un temps fort, plutôt comme un contrepoint. Cependant, un élément nouveau de ces pièces apporte une ponctuation différente dans le temps dramatique: ballades et complaintes dramatiques représentent un silence de l'action qui se trouve suspendue pour un temps plus ou moins long, mais pendant lequel, en fait se passent les choses essentielles, car la complainte se place au cour d'un rituel incantatoire.

Si nous prenons comme exemple deux complaintes de périodes différentes, nous pouvons voir ce qui les unit, et quelles fonctions elles remplissent au sein des pièces. Il s'agit de la complainte de Pithias dans Damon and Pithias de Richard Edwards $(1564)^{4}$, et celle de Panthea dans The Wars of Cyrus complainte composée par Richard Farrant et pour laquelle l'in-4to de 1594 ne donne aucune indication scénique, mais que l'on trouve dans plusieurs manuscrits musicaux de la période 5 .

L'analyse des procédés verbaux et musicaux utilisés révèle qu'il s'agit d'une forme fortement ritualisée. Allitérations, invocations des puissances supérieures, appel réitéré du nom de l'être aimé disparu, tous ces procédés sont présents dans les complaintes, depuis leur apparition dans le théâtre Tudor. 
Lorsque Damon est condamné à mort, son ami Pithias, se trouvant avec lui en terre étrangère, ne sait vers qui se tourner. L'émotion atteint son paroxisme d'intensité alors qu'il mesure l'injustice de la condamnation, et son impuissance à y remédier. Il faut ici une interruption de l'action, une parenthèse, un silence qui permettra d'y voir clair ; la complainte nous projette, ainsi que le personnage qui la chante, dans un temps qui n'est plus celui de l'action, mais celui de l'émotion, un temps où celui-ci peut librement s'épancher, pour faire ensuite place à l'action positive. Pithias en appelle alors à tous les infortunés, "Awake ye woful wights ", qu'il prend à témoin, et se fait porteur de leur douleur, "resign to me your plaints and tears"; puis il invoque les furies, et songe lui même à la mort :

O Death come now, seeing I hear

Damon my friend must die.

Ici, le refrain reprenant le nom de Damon, produit l'effet incantatoire désiré, en s'ajoutant aux nombreuses allitérations.

Pour Pantheas, l'issue diffère, car son mari est bel et bien mort. Son désespoir s'exprime également par une invocation des puissances suprêmes, "Alas ye salt sea Gods, bowe down your eares devine ". Le nom de l'être aimé disparu, est répété, la mort invoquée, * come death alas, O Death most sweet / for nowe, for nowe, for nowe I crave to die, to die, to die, to die ". A la différence de Pithias, Panthea, elle, meurt en effet. La complainte l'élève au rang des infortunées tragiques, Phèdre, Didon... Au moment où elle chante, l'héroīne déjà n'est plus, elle n'appartient plus au temps de la réalité, ni à celui de l'action de la pièce, mais rejoint d'une certaine façon, la notion d'éternité.

Une utilisation similaire, mais beaucoup plus subtile de la complainte nous est offerte par Shakespeare, avec la ballade de Desdémone. L'action se tait pour faire place au chant, point d'orgue de l'action, pendant lequel on entend encore la musique qui précède, et l'on anticipe celle qui va suivre. La complainte de Desdémone, née des événements qui la précèdent, annonce aussi sa mort.

Comme l'épisode central des "Wit plays", la complainte nous transporte dans un point du temps où tous les temps sont réunis, dont celui de la mort. C'est ce sens tout particulier que prend l'élégie, 
forme musicale dont s'inspirent très clairement les complaintes dramatiques. Je voudrais donner ici comme exemple l'élégie sur la mort de Thomas Tallis, de William Bird, qui, nous le savons, collabora, en tant que musicien, à l'élaboration de pièces de théâtre, pour lesquelles il composa justement des complaintes. En voici le texte :

Ye sacred Muses, race of Jove, whom Music's lore delighteth Come down, come down from crystal heavens above To earth where sorrow dwelleth, In mourning weeds, in mourning weeds, with tears in eyes. Talis is dead, Tallis is dead, and Music dies.

Invocation des Muses, répétition du nom de Tallis, association de l'ensemble de la musique à la mort du musicien, ces effets se trouvent accentués par une mise en musique suivant de près le sens même du texte. On remarque tout particulièrement les intervalles descendants sur "come down", et la montée mélodique sur "from heavens above", ou encore la formule mélismatique qui laisse le texte en suspens sur " Music dies ».

Audition: "Ye Sacred Muses ». Chant: Francis Guinle, hautecontre ; clavecin : Sylvie Lannes 6 .

Ces complaintes représentent deux aspects du rite dramatique : d'une part, un arrêt de l'action pour apporter un commentaire ou une justification, en purgeant les émotions qui sont un obstacle à l'action ; d'autre part, par la suspension même du temps de la narration, elles placent les personnages et les spectateurs dans un temps où une vision de l'éternel et du divin devient possible. Elles marquent ainsi un tournant décisif, et après elles, le monde ne peut plus être le même.

Cette fonction de la complainte est reprise plus tard par un autre aspect de l'élément musical: le rôle magique de la musique qui l'associe souvent à la musique des sphères, moment où l'harmonie divine prend le pas, et qui correspond aussi à un silence de l'action, moment hors du temps, et où tout, en fait, se passe.

Ainsi, dans Le Conte d'Hiver lorsque Leontes retrouve sa femme, et que, au son de la musique, la statue d'Hermione se met en mouvement, cette suspension d'un instant représente un retour en arrière 
de seize ans. De même lorsque Périclès retrouve sa fille, et revient à la vie, la musique des sphères se fait entendre, promettant un retour à l'harmonie qui régnait avant que la discorde ne s'installe : au début de la pièce, après sa découverte de l'inceste, Périclès compare la fille d'Antiochus à une viole :

You are a fair viol and your sense the strings, Who, fingered to make man his lawful music, Would draw heaven down and all the gods to hearken, But being played upon before your time, Hell only danceth at so harsh a chime.

(Acte I, scène $1,11.81-85)^{7}$

Dans les deux pièces il s'agit bien d'un trajet initiatique, comme dans les moralités, et comme dans Damon and Pithias, conservant un aspect allégorique sous l'apparence narrative.

Le cas présenté par Périclès est tout à fait clair. Dans un monde où règne la discorde, soulignée par la métaphore de la viole, la fuite de Périclès représente le départ d'un voyage qui utilise à la fois le temps de la réalité (passage des saisons, mariage du protagoniste, naissance et éducation de Marina...) et le temps ritualisé du trajet initiatique, marqué par des temps forts où alternent confiance et trahison, comme autant d'épreuves infligées, avant d'atteindre l'harmonie finale.

Le couple formé par Antiochus et sa fille sonne comme un contrepoint infernal au couple Périclès-Marina, pris dans l'harmonie céleste de la fin de la pièce. Par son vice, la fille d'Antiochus provoquait la discorde, par sa vertu, Marina déclenche le processus du retour à l'harmonie. La chute, ou la mort métaphorique subie par le protagoniste, constitue une des étapes essentielles du trajet initiatique ; Périclès s'en relève et revient à la vie, et ce retour s'accompagne de musique, et s'effectue par son intermédiaire. Soudain tout semble rester en suspens, alors que se fait entendre, à lui seul, la musique des sphères :

Pericles : But, what music?

Helicanus : My lord I hear none.

Pericles : None?

The music of the spheres! List, my Marina. 
Lysimacus : It is not good to cross him ; give him way.

Pericles : Rarest sounds! Do ye not hear?

Lysimacus : Music, my lord?

Pericles : I hear most heavenly music.

It nips me unto listning, and thick slumber

Hangs upon mine eyes : let me rest.

(Acte $\mathrm{V}$, scène 1, 1 1. 231-239.)

Il s'endort, alors que Diane lui apparaît et lui indique le moyen de retrouver sa femme.

Au-delà du temps de la réalité, d'une longue période de la vie de Périclès, le temps qui fait fonctionner la pièce est celui-là même des moralités : il s'agit du temps du passage de la discordance à l'harmonie, du désordre à l'ordre, il s'agit, pour le protagoniste, du temps du salut.

\section{Francis GUINLE}

\section{NOTES}

1) Wilfrid Mellers, Harmonious Meetings, a Study of the Relationship between English Music, Poetry and Theatre (Londres : Dennis Dobson, 1965).

2) La référence est à l'édition Malone Society Reprints, La pièce est de Richard Wever.

3) Wit and Science (John Redford, 1539) The Marriage of Wit and Science (1568), A Contract of Marriage between Wit and Wisdom (Francis Merbury, 1579).

4) Pour les dates, je suis les indications données par Harbage, Annuals of English Drama, 975-1700, revised by S. Schoenbaum (Londres: Methuen, 1964). On trouvera la complainte de Pithias à la page 584 de l'anthologie de J. Q. Adams, Chief Pre-Shakespearean Dramas (Cambridge Massachusetts : 1924).

5) On pense que la pièce publiée est en fait reprise d'une pièce antérieure, c. 1578, de Richard Farrant; pour les détails sur l'attribution, voir l'édition de J. P. Brawner, University of Illinois Studies in Language and Literature, 28, n ${ }^{\circ} 3-4$ (Urbana, 1942). La musique se trouve dans trois manuscrits, diversement attribuée: B. L. Add. MSS. 17786-17791 ; Oxford, Christ Church, Music MSS 984-988; et Tenbury MS. 389.

6) Il s'agit, en fait, d'une composition pour voix et quatuor de violes, selon la tradition des "consort songs". J'ai fait une réduction des parties de violes pour clavecin.

7) Références à l'édition de John Dover Wilson et Arthur Quiller Couch, The New Shakespeare (Cambridge University Press, 1957). 